\title{
High Performance Asphalt Pavements
}

\author{
Peter E. Sebaaly \\ University of Nevada \\ USA \\ psebaaly@unr.edu
}

\begin{abstract}
Asphalt pavements make up 95 percent of the road network throughout the world. Typical service life of an asphalt pavement is between 15 and 20 years. Under the combined actions of traffic loads and environment, asphalt pavements experience multiple modes of distresses, including; rutting, cracking, raveling, and bleeding. This presentation will cover recent advances in methods of pavement analysis, materials selection, modifications, and additives that make it possible to extend the service life of asphalt pavements to 25+ years. Pavement analysis that takes into consideration the dynamic nature of traffic loads and accounts for the shear stresses imposed by braking of heavy vehicles allows the design engineer to accurately identify the applied loads and recommend the appropriate pavement structure to resist these loads. The materials selection process is very critical in order to match the properties of the materials with the combined demand of traffic loads and environment. Selecting inadequate materials for the asphalt mix will lead to very early failures of the pavement in terms of rutting and bleeding while selecting very stiff and bad-aging materials will lead to long-term failures in terms of cracking. However, if high quality materials are not locally available, the design engineer has the access to various modification techniques, including; polymer and ground tire rubber. These modifiers improve the performance characteristics of asphalt mixtures while reducing the burden of waste products on the society. Finally, several additives are also available to improve the resistance of the asphalt pavement to moisture damage which is the most significant factor in reducing service life. These advances increase the resistance of the asphalt pavement to the various distresses, which results in extended service life at a significantly lower life cycle cost.
\end{abstract}

